\title{
GLII and CTNNB1 Knockdown Activates NOTCH and mTOR Signalling in NB4 Myeloid Leukaemia Cells
}

\author{
YUKI OKUHASHI $^{1,2}$, MAI ITOH ${ }^{1}$ and SHUJI TOHDA ${ }^{1}$ \\ ${ }^{I}$ Department of Laboratory Medicine, Tokyo Medical and Dental University, Tokyo, Japan; \\ ${ }^{2}$ Department of Medical Technology, Tokyo University of Technology, Tokyo, Japan
}

\begin{abstract}
Background: Hedgehog (HH), WNT, NOTCH, and mechanistic target of rapamycin (mTOR) signalling pathways are known to regulate the progression of cancer; however, their interaction in leukaemia cells is not fully clarified. Materials and Methods: Myeloid and T-lymphoblastic leukaemia cell lines (NB4, THP-1, Jurkat, and DND-41) were transfected with small interfering RNAs targeting the gliomaassociated oncogene homolog 1 (GLII) and catenin beta-1 (CTNNB1) genes involved in the regulation of $\mathrm{HH}$ and WNT pathways, respectively, and we examined cell proliferation and gene expression. Results: The knockdown of GLII and CTNNB1 did not significantly affect proliferation of any cell line; however, it up-regulated the expression of NOTCH1, cleaved NOTCHI fragment, and phosphorylated mTOR in NB4 cells, but not in the other cell lines. Conclusion: Our data suggest that HH and WNT act upstream of NOTCH and mTOR pathways and negatively regulate them in myeloid NB4 cells. Further studies are required to determine the biological significance of this signalling crosstalk in leukaemia.
\end{abstract}

Hedgehog $(\mathrm{HH})$ and WNT signalling pathways are known to play an important role in regulating cell proliferation and differentiation (1-4). When HH proteins are bound to their receptor Patched 1, glioma-associated oncogene homolog (GLI) is translocated into the nucleus, where it regulates the transcription of target genes involved in cell growth (5-8). However, the effects of $\mathrm{HH}$ ligands in leukaemia cells have not been fully elucidated.

WNT proteins activate the canonical WNT signalling cascade by inducing nuclear translocation of $\beta$-catenin and regulating gene expression. WNT proteins also activate the

Correspondence to: Shuji Tohda, MD, Department of Laboratory Medicine, Tokyo Medical and Dental University, Yushima 1-5-45, Bunkyo-Ku, Tokyo 113-8519, Japan. Tel: +81 358035334; Fax: +81 358035629; e-mail: tohda.mlab@tmd.ac.jp

Key Words: Hedgehog, WNT, NOTCH, siRNA, leukaemia. non-canonical WNT signalling cascade, which is independent of nuclear translocation of $\beta$-catenin $(9,10)$.

Previously, we reported that $\mathrm{HH}$ inhibitor cyclopamine and WNT inhibitor quercetin suppressed the growth of leukaemia cells $(11,12)$; however, these compounds have off-target effects. We also showed that small interfering (si)RNA-mediated knockdown of NOTCH1 and NOTCH2 suppressed the growth of T-cell acute lymphoblastic leukaemia (T-ALL) cell lines (13), suggesting that specific inhibition of HH and WNT pathways with siRNAs might be an effective approach to investigating the role of $\mathrm{HH}$ and WNT signalling in leukaemia cells.

The interplay among HH, WNT, NOTCH, and mechanistic target of rapamycin (mTOR) pathways is known to regulate cell stemness (14); however, their relationship in leukaemia should be clarified. To address this question, in this study, the expression of GLII and catenin beta-1 (CTNNBI) gene, which mediate HH and WNT signalling, respectively, were knockeddown in acute myeloid leukaemia (AML) and T-ALL cell lines and the effects on cell proliferation and NOTCH and mTOR pathways were assessed.

\section{Materials and Methods}

Cell culture and siRNA transfection. Two AML cell lines (NB4 and THP-1) and two T-ALL cell lines (Jurkat and DND-41) were used. NB4 derived from a patient with acute promyelocytic leukaemia was kindly provided by Dr. M. Lanotte (France), THP-1 derived from a patients with acute monocytic leukaemia was obtained from the Japanese Cancer Research Resources Bank, and Jurkat and DND-41 cells were donated by Drs. Harashima and Orita (Fujisaki Cell Centre, Japan). All cells were cultured in RPMI-1640 supplemented with $10 \%$ foetal calf serum (FCS).

Three sets of pre-designed siRNAs targeting GLII (siGL: HSS104170, HSS178441, and HSS178442), CTNNB1 (siCT: HSS102460, HSS102461, and HSS102462), and NOTCH1 (siN1: HSS 107248, HSS107249, and HSS181550) were purchased from Life Technologies (Carlsbad, CA, USA). Stealth RNAi negative control Duplex was used as a control siRNA (siCont). Cells $\left(7 \times 10^{5}\right.$ per $10-\mu \mathrm{l}$ tip) were transfected with $40 \mathrm{nM}$ of each siRNA using the Neon $^{\mathrm{TM}}$ Transfection system (Life Technologies) according to the manufacturer's instructions, and immediately transferred to cell culture medium. 
Quantitative reverse transcription-polymerase chain reaction ( $R T$ $P C R$ ). Cells transfected with siRNAs were cultured for $24 \mathrm{~h}$ and mRNA expression of GLII, CTNNB1, and NOTCH1 was measured by quantitative RT-PCR using the FastStart DNA Master SYBR Green I kit with LightCycler primer sets (Roche Diagnostics, Mannheim, Germany). Expression of the target genes was normalized to that of the $\beta$-actin-encoding gene (ACTB) and presented as the mean percentage of expression in siCont-transfected cells.

Cell growth assay. Short-term cell growth was examined using a colorimetric WST-8 assay (Dojindo Laboratories, Kumamoto, Japan). Cells transfected with siRNAs were plated in $0.1 \mathrm{ml}$ of $10 \%$ FCSsupplemented RPMI-1640 in 96-well culture plates $\left(5 \times 10^{4}\right.$ cells/well) and cultured for 3 days. WST-8 [2-(2-methoxy-4-nitrophenyl)-3-(4nitrophenyl)-5-(2,4-disulfophenyl)- $2 H$-tetrazolium, monosodium salt] was then added to the wells and optical density (OD) was measured in an enzyme-linked immunosorbent assay plate reader. Cell growth was expressed as the mean percentage of the OD value for siConttransfected cells.

Immunoblotting. Cells transfected with siRNAs were harvested 2 days later and lysed. Cell lysates were subjected to sodium dodecyl sulphate-polyacrylamide gel electrophoresis (SDS-PAGE) and immunoblotting with antibodies against GLI1, phospho- $\beta$-catenin (Ser675), $\beta$-catenin, NOTCH1, cleaved NOTCH1 (Val1744), MYC, and phospho-mTOR (Ser2481) (Cell Signaling Technology, Danvers, MA, USA); anti- $\alpha$-tubulin antibody (Abcam, Cambridge, MA, USA) was used to ensure equal loading. Three independent experiments were performed to verify reproducibility. Qualitative assessment of the immunoblot assay was performed by comparing the density of bands from the target siRNA-transfected cells and control siRNA-transfected cells.

Statistical analysis. The data are expressed as the mean \pm standard deviation (SD). Student's $t$-test was used to determine statistical significance of the differences between siCont- and target siRNAtreated cells; values of $p<0.05$ was considered significant.

\section{Results}

Effects of siRNAs on GLI1, CTNNB1, and NOTCH1 expression. The most potent among siGL siRNAs were HSS104170 for T-ALL and HSS178441 for AML cell lines; among siCT siRNAs, HSS102460 for all cell lines, and among siN1 siRNAs, HSS107248 for T-ALL and HSS107249 for AML cell lines. The knockdown effects of these siGL, siCT, and siN1 siRNAs on the expression of GLII, CTNNB1, and NOTCH1 mRNA are shown in Table I. Transfection with siGL, siCT, and siN1 selectively suppressed the mRNA expression of GLI1, CTNNB1, and NOTCH1, respectively.

Effect of GLII and CTNNBI knockdown on short-term cell growth. The results indicated that the knockdown of GLII and $C T N N B 1$ genes had no significant effects on short-term cell proliferation (Figure 1).

Effects of GLI1, CTNNB1, and NOTCH1 knockdown on the expression of signalling proteins. Figure 2 shows the results of
Table I. Efficiency of glioma-associated oncogene homolog 1 (GLI1), catenin beta-1 (CTNNB1), and NOTCH1 knockdown by specific siRNAs. The numbers in siCont columns indicate the ratio obtained by dividing the expression level of each mRNA by that of $\beta$-actin mRNA in control siRNA (siCont)-transfected cells. Effects of specific siRNAs on gene expression are presented as the mean percentage of that in siConttransfected cells.

\begin{tabular}{lcccccc}
\hline & \multicolumn{2}{c}{ GLI1 } & \multicolumn{2}{c}{ CTNNB1 } & \multicolumn{2}{c}{ NOTCH1 } \\
\cline { 2 - 7 } Cell line & siCont & siGL & siCont & siCT & siCont & siN1 \\
\hline NB4 & $3.01 . \mathrm{E}-03$ & $24.2 \%$ & $5.71 . \mathrm{E}-02$ & $27.5 \%$ & $4.59 . \mathrm{E}-01$ & $24.6 \%$ \\
THP-1 & $4.60 . \mathrm{E}-01$ & $48.0 \%$ & $5.56 . \mathrm{E}-01$ & $19.8 \%$ & $6.32 . \mathrm{E}-02$ & $9.3 \%$ \\
Jurkat & $1.12 . \mathrm{E}-02$ & $51.7 \%$ & $3.11 . \mathrm{E}-02$ & $30.8 \%$ & $1.70 . \mathrm{E}-01$ & $30.5 \%$ \\
DND-41 & $1.20 . \mathrm{E}-02$ & $17.0 \%$ & $1.88 . \mathrm{E}-02$ & $26.9 \%$ & $8.59 . \mathrm{E}-02$ & $47.6 \%$ \\
\hline
\end{tabular}

protein expression analysis for NB4 and Jurkat chosen as representative cell lines. Transfection with siN1, siGL, and siCT suppressed the expression of NOTCH1, GLI1, and $\beta$ catenin proteins in NB4 and Jurkat cells. Interestingly, GLII and CTNNB1 knockdown in NB4 cells increased the expression levels of NOTCH1, cleaved NOTCH1 fragment (active form of NOTCH1), and phosphorylated mTOR, whereas NOTCH1 knockdown slightly increased phosphorylated mTOR (Figure 2, left panel). However, no such effects on protein expression were observed in transfected Jurkat cells (Figure 2, right panel) nor in DND-41 and THP-1 cells (data not shown). The combined transfection with siGL and siCT siRNAs did not increase the up-regulatory effects on protein expression in NB4 cells (Figure 2, left panel).

\section{Discussion}

The results of our siRNA-mediated knockdown experiments indicate that $\mathrm{HH}$ and canonical WNT signalling did not significantly affect proliferation of the four leukaemia cell lines. Interestingly, we found that GLII and CTNNBI knockdown up-regulated the expression of NOTCH1, cleaved NOTCH1 fragment, and phosphorylated mTOR in NB4 cells. These findings indicate that inhibition of WNT and $\mathrm{HH}$ signalling activates NOTCH and mTOR pathways in some AML cells, suggesting a possibility that WNT and $\mathrm{HH}$ function upstream of NOTCH and mTOR pathways (Figure 3). The knockdown of NOTCHI in NB4 cells did not inhibit mTOR phosphorylation, suggesting that mTOR signalling is not located downstream of NOTCH1. However, these effects were not observed in the other cell lines.

Previous studies investigating the relationship among $\mathrm{HH}$, WNT, NOTCH, and mTOR pathways found that NOTCH receptor activation induced $\mathrm{HH}$ and mTOR signalling (15) and that the NOTCH pathway positively regulated mTOR phosphorylation (16), whereas mTOR directly activated GLI1 


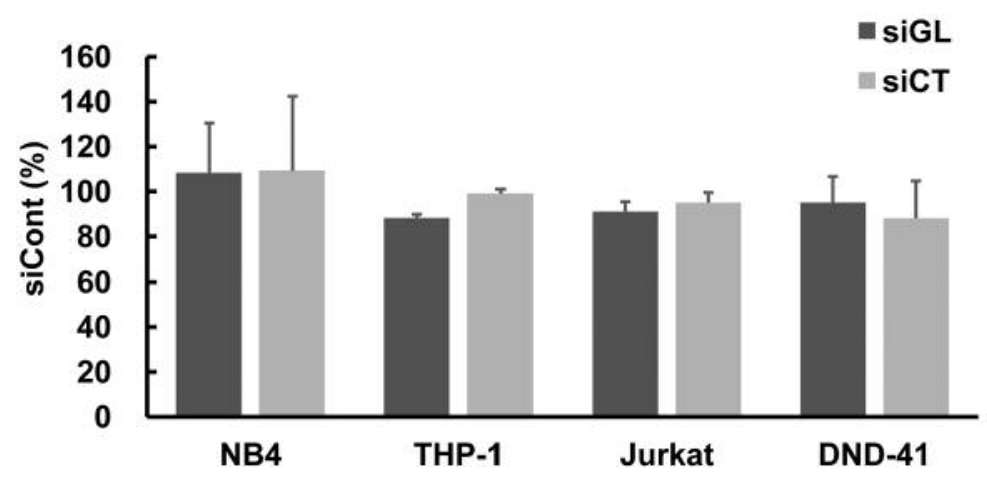

Figure 1. Effect of glioma-associated oncogene homolog 1 (GLI1) and catenin beta-1 (CTNNB1) knockdown on short-term cell growth. Leukaemia cells were transfected with siRNAs targeting GLII (siGL) or CTNNB1 (siCT) and analyzed for proliferation after 3 days using a colorimetric assay. Cell growth is presented as the percentage of the mean OD value normalized to that of control siRNA (siCont)-transfected cells.

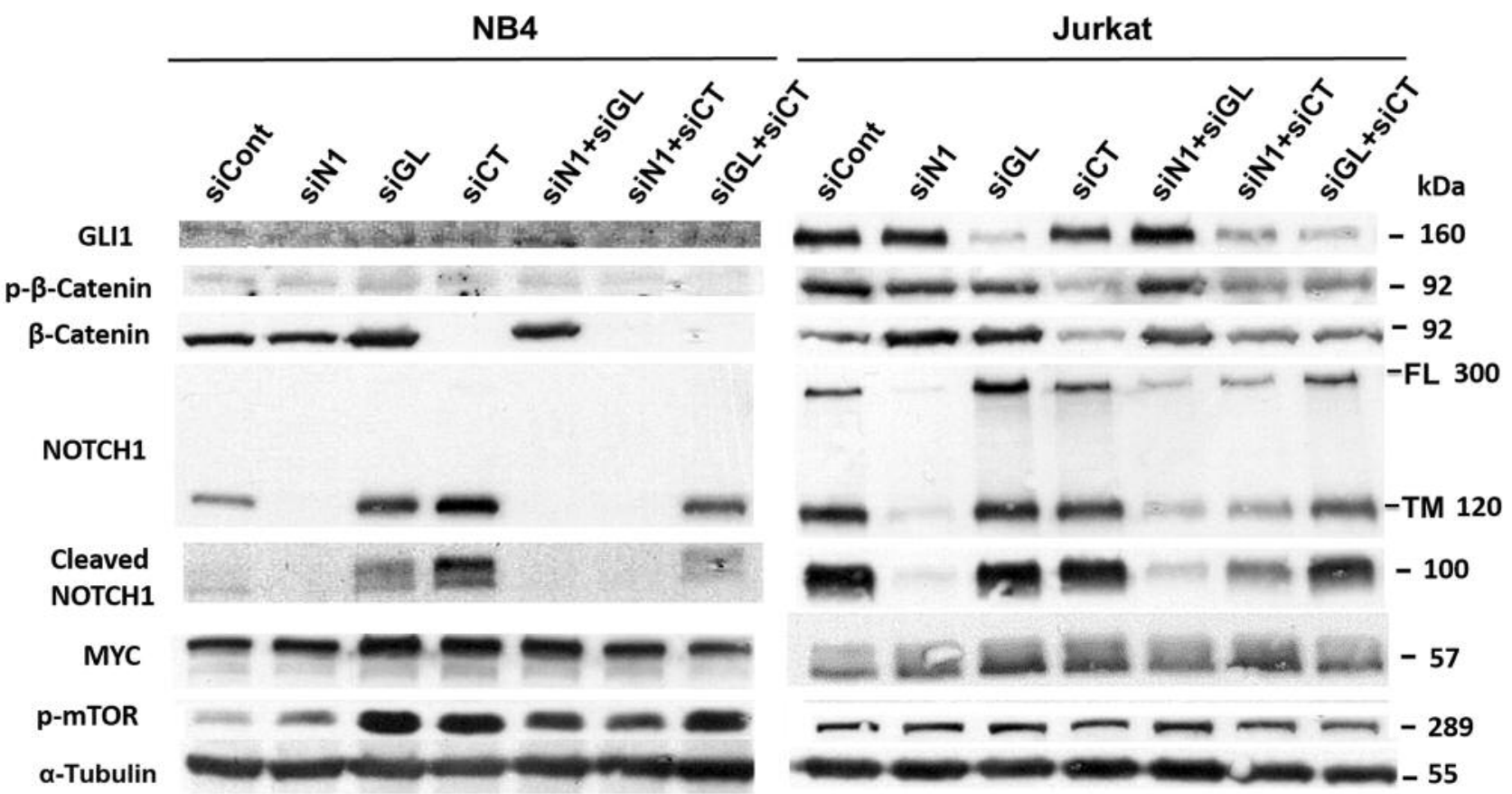

Figure 2. Effects of glioma-associated oncogene homolog 1 (GLI1), catenin beta-1 (CTNNB1), and NOTCH1 silencing on the expression of signalling proteins. Cells were transfected with the indicated siRNAs or their combinations for $24 \mathrm{~h}$ and analyzed for protein expression by immunoblotting. FL: Full-length NOTCH1; p-: phosphorylated; TM: transmembrane subunit of NOTCH1.

and smoothened (SMO), effectors of the Sonic HH pathway, in oesophageal adenocarcinoma (17). Furthermore, WNT3A induced the expression of GLI1 and GLI3 and Sonic HH transcription in patients with chronic myeloid leukaemia (18). These studies suggest that NOTCH regulates mTOR and $\mathrm{HH}$ signalling and acts upstream of these pathways.

Thus, our study revealed a novel mode of interaction among the four signalling cascades; however, it had limitations. Firstly, the novel relationship was observed only in one out of the four cell lines investigated; secondly, gene silencing by siRNAs might be too weak to be translated to the cellular level; and thirdly, the underlying molecular mechanisms and significance of the observed effect have not been elucidated. Further experiments are needed to clarify the functional role of the proposed crosstalk among $\mathrm{HH}$, WNT, NOTCH, and mTOR pathways in leukaemia, which 


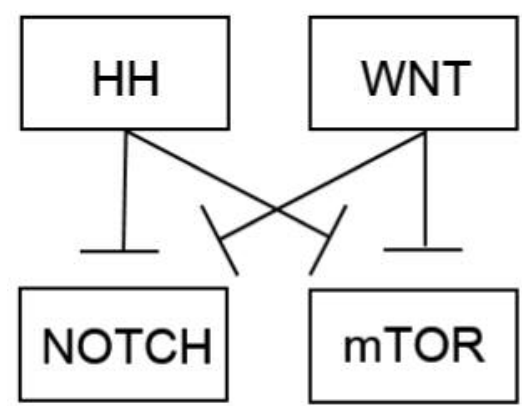

Figure 3. Schematic representation of a possible crosstalk among Hedgehog (HH), WNT, NOTCH and mechanistic target of rapamycin (mTOR) signalling pathways in NB4 cells.

may promote the development of HH- and WNT-targeting therapeutic approaches.

\section{Acknowledgements}

This work was supported, in part, by a Grant-in-Aid for Young Scientists (B) from the Japan Society for the Promotion of Science (No. 16K19205).

\section{References}

1 Freestone SH, Marker P, Grace OC, Tomlinson DC, Cunha GR, Harnden $\mathrm{P}$ and Thomson AA: Sonic hedgehog regulates prostatic growth and epithelial differentiation. Dev Biol 264: 352-362, 2003.

2 Ingham PW and Placzek M: Orchestrating ontogenesis: Variations on a theme by sonic hedgehog. Nat Rev Genet 7: 841850, 2006.

3 Reya T, Duncan AW, Ailles L, Domen J, Scherer DC, Willert K, Hintz L and Nusse R: A role for WNT signaling in self-renewal of haematopoietic stem cells. Nature 423: 409-414, 2003.

4 Willert K, Brown JD, Danenberg E, Duncan AW, Welssman IL, Reya T, Yates JR III. and Nusse R: WNT proteins are lipidmodified and can act as stem cell growth factors. Nature 423: 448-452, 2003.

5 Lee J, Platt KA, Censullo P, Ruiz I and Altaba A: Gli1 is a target of Sonic hedgehog that induces ventral neural tube development. Development 124: 2537-2552, 1997.

6 Chen JK, Taipale J, Cooper MK and Beachy PA: Inhibition of Hedgehog signaling by direct binding of cyclopamine to Smoothened. Genes Dev 16: 2743-2748, 2002.
7 Zhao C, Chen A, Jamieson CH, Fereshteh M, Abrahamsson A, Blum J, Kwon HY, Kim J, Chute JP, Rizzieri D, Munchhof M, VanArsdale T, Beachy PA and Reya T: Hedgehog signalling is essential for maintenance of cancer stem cells in myeloid leukemia. Nature 9: 776-779, 2009.

8 Jia Y, Wang Y and Xie J: The Hedgehog pathway: Role in cell differentiation, polarity and proliferation. Arch Toxicol 89: 179191, 2015.

9 MacDonald BT, Tamai K and He X: WNT/ $\beta$-catenin signaling: components, mechanisms, and diseases. Dev Cell 17: 9-26, 2009.

10 Reya T and Clevers H: WNT signalling in stem cells and cancer. Nature 434: 843-850, 2005.

11 Kawahara T, Kawaguchi-Ihara N, Okuhashi Y, Itoh M, Nara N and Tohda S: Cyclopamine and quercetin suppress the growth of leukemia and lymphoma cells. Anticancer Res 29: 4629-4632, 2009.

12 Okuhashi $\mathrm{Y}$, Itoh $\mathrm{M}$, Nara $\mathrm{N}$ and Tohda $\mathrm{S}$ : Effects of combination of NOTCH inhibitor plus hedgehog inhibitor or WNT inhibitor on growth of leukemia cells. Anticancer Res 31: 893-896, 2011.

13 Okuhashi Y, Itoh M, Nara N and Tohda S: NOTCH knockdown affects the proliferation and mTOR signaling of leukemia cells. Anticancer Res 33: 4293-4298, 2013.

14 Mimeault M and Batra SK: Novel biomarkers and therapeutic targets for optimizing the therapeutic management of melanomas. World J Clin Oncol 3: 32-42, 2012.

15 Androutsellis-Theotokis A, Leker RR, Soldner F, Hoeppner DJ, Ravin R, Poser SW, Rueger MA, Bae SK, Kittappa R and McKay RD: NOTCH signalling regulates stem cell numbers in vitro and in vivo. Nature 442: 823-826, 2006.

16 Chan SM, Weng AP, Tibshirani R, Aster JC and Utz PJ: NOTCH signals positively regulate activity of the mTOR pathway in Tcell acute lymphoblastic leukemia. Blood 110: 278-286, 2007.

17 Wang Y, Ding Q, Yen CJ, Xia W, Izzo JG, Lang JY, Li CW, Hsu JL, Miller SA, Wang X, Lee DF, Hsu JM, Huo L, Labaff AM, Liu D, Huang TH, Lai CC, Tsai FJ, Chang WC, Chen $\mathrm{CH}$, Wu TT, Buttar NS, Wang KK, Wu Y, Wang H, Ajani J and Hung MC: The crosstalk of mTOR/S6K1 and Hedgehog pathways. Cancer Cell 21: 374-387, 2012.

18 Sengupta A, Banerjee D, Chandra S, Banerji SK, Ghosh R, Roy $\mathrm{R}$ and Banerjee $\mathrm{S}$ : Deregulation and cross talk among Sonic hedgehog, WNT, HOX and NOTCH signaling in chronic myeloid leukemia progression. Leukemia 21: 949-955, 2007.

Received October 2, 2018

Revised October 15, 2018

Accepted October 16, 2018 